\title{
Especies pioneras, persistentes y ensayos de germinación en bosques montanos de la cordillera central, colombia
}

\author{
Pioneer Species, persistents and germination tests \\ in montane forests of Central Ridge, Colombia
}

Fecha de Recepción: 19.02 .2020

Fecha de Aceptación: 05.05.2020

Doi: https://doi.org/10.19053/01217488.v11.n2.2020.10645

\section{Resumen}

La alta fragmentación y deterioro de los bosques montano bajo hace prioritaria su restauración ecológica, debido a esto, se requieren mecanismos que propendan a la recuperación del bosque, sobre todo en áreas prioritarias. El presente estudio pretende aportar herramientas que permitan a los restauradores tener información sobre especies nativas con potencial para la restauración. Para el efecto, se seleccionó un bosque húmedo montano bajo de 500 ha, el cual, se dividió en tres zonas de acuerdo a su intervención antrópica. En cada zona se realizaron análisis físico-químicos de suelos e inventarios florísticos analizados con diferentes índices de diversidad y perfiles de vegetación. Además, se clasificaron las especies arbóreas y arbustivas en pioneras y persistentes. Finalmente, se colectaron y analizaron frutos y semillas de las especies que se encontraban fructificadas al momento del muestreo, realizando pruebas de germinación en cada uno de ellos. Los resultados sugieren que en general las zonas presentaron diversidad alta (índice Shannon) con valores entre 2.804 y 3.414. Las especies con mayor IVI fueron Miconia resima, Palicourea angustifolia, Palicourea garciae, Cavendishia pubescens, y Clusia multiflora. La mayor cantidad de especies fructificadas se encontró en las áreas 1 y 2 (intervenida y moderadamente intervenida), el $40 \%$ de las semillas extraídas germinaron, finalmente, se clasificaron 42 especies en las categorías de pioneras y persistentes. En conclusión, se espera que la información obtenida en el presente proyecto sirva en parte como herramienta de apoyo para la restauración ecológica del bosque húmedo montano bajo.

Palabras clave: Bosque húmedo montano bajo, diversidad, grupos funcionales, restauración ecológica.

\begin{abstract}
High fragmentation and deterioration of the low montane humid forest makes its ecological restoration a priority, due to this, mechanisms that tend to recover the forest are required, especially in priority areas. This study aimed to provide tools that allow restorers to have information about native species with potential for restoration. For this purpose, a 500-hectare low montane humid forest was selected, which was divided into three zones according to its anthropic intervention. In each area physicochemical analyzes of soils and floristic inventories were carried out analyzed with different indexes of diversity and vegetation profiles. In addition, tree and shrub species were classified as pioneer and persistent. Finally, fruits and seeds of the species that were fructified at the time of sampling were collected and analyzed, carrying out

a Docente Asociado, grupo de Estudios Florísticos, programa Ingeniería Ambiental, Facultad de Ingenierías, Universidad Católica de Oriente. jsierra@uco.edu.co

b Director del grupo de estudios florísticos, programa de Ingeniería Ambiental, Facultad de Ingeniería. Universidad

Católica de Oriente.maquijano@uco.edu.co

Estudiante de biología, Universidad de Antioquia.

Estudiante de Ingeniería Ambiental, Universidad Católica de Oriente.
\end{abstract}


germination tests in each of them. The results suggest that in general the zones presented high diversity (Shannon index) with values between 2.804 and 3.414. Species with the highest IVI were Miconia resima, Palicourea angustifolia, Palicourea garciae, Cavendishia pubescens, and Clusia multiflora. The largest number of fruiting species was found in areas 1 and 2 (intervened and moderately intervened), $40 \%$ of the seeds extracted germinated, finally, 42 species were classified in the pioneer and persistent categories. In conclusion, the information obtained in this project is expected to serve in part as a support tool for the ecological restoration of the low montane humid forest.

Key words: Low montane humid forest, diversity, functional groups, ecological restoration.

\section{INTRODUCCIÓN}

El planeta posee 3,682 millones de hectáreas de bosque natural, de las cuales el $4 \%$ pertenece a bosques de montaña, y el $60 \%$ de estas se ubican en centro y sur América. Los bosques montanos y montanos bajos se encuentran distribuidos a lo largo del territorio latinoamericano y del Caribe. Son muchos los motivos que corroboran la importancia de estos bosques, uno de los principales radica en la protección crucial de las cuencas hidrográficas, ya que captan y regulan grandes cantidades de agua. Estos bosques debido a su característica nubosa adicionan generalmente entre $5 \%$ y $20 \%$ del volumen normal de lluvias. Además, su alta diversidad y endemismos es otra característica que los resalta [1].

Desafortunadamente, todos estos bosques han sido transformados y fragmentados, esto es asociado con procesos progresivos de pérdida de hábitat causados por el mal uso del territorio. La transformación y la reducción en el tamaño de las coberturas de bosque dan lugar a diferentes efectos negativos como: la pérdida de hábitat y disminución del tamaño de las poblaciones de fauna y flora, aumento en la temperatura de la superficie del suelo, disminución en la evapotranspiración y precipitación, lo que conlleva a la alteración de múltiples procesos ecológicos [2], [3]. Adicional a esto, la mayoría de los fragmentos de bosque húmedo montano bajo (BHMB) que aún quedan, son sometidos a quemas, extracción de leña, maderas y especies ornamentales nativas, lo que genera anomalías en su diversidad, y cambios en su composición [4].

En Colombia, los BHMB se encuentran ubicados en la región Andina donde se presenta el mayor asentamiento humano del país, y es ahí donde predominan muchas de las áreas degradadas con evidencias de los problemas antes mencionados [1], [5]. Para restaurar parte de estas áreas degradadas, el gobierno de Colombia firmó el Desafío de Bonn, con el compromiso de hacer restauración ecológica de un millón de hectáreas para el año 2020 [6]. Para cumplir con este compromiso, es muy importante desarrollar herramientas que propendan por una restauración ecológica exitosa. Saber cuáles especies se deben seleccionar en la restauración ecológica, es una de las preguntas cruciales que se deben responder en este tipo de iniciativas. En algunos casos se han cometido muchos errores en este tipo de selección, lo cual conlleva a procesos de reforestación que no implican necesariamente restauración. Generar herramientas que ayuden en esta selección sería de gran importancia para los investigadores y técnicos. Es necesario, buscar opciones de restauración ecológica donde se hace uso de los recursos naturales o del ambiente, a fin de imitar la estructura, función, diversidad y dinámica del ecosistema específico [7]. Además de estas consideraciones, para la restauración ecológica, se deben tener en cuenta, los pisos térmicos, las características físico-químicas, ambientales, y múltiples zonas de vida que son propias de los diversos ecosistemas de Colombia [8].

Estos cambios de las condiciones naturales en los fragmentos de BHMB, afectan directamente los bancos y lluvias de semillas nativas, debido a la erosión del suelo, mayor depredación de semillas, condiciones climáticas extremas, baja viabilidad de propágulos, y mayor competencia con pastos [9]. Para algunos autores, la baja cantidad de bancos y lluvias de semillas, es una de las mayores limitaciones para la regeneración de los bosques [10], [11]. Estas son algunas de las razones por las cuales es importante conocer las dinámicas de frutos y semillas de los remanentes de BHMB que aún quedan. Infortunadamente, en la actualidad, en estos bosques, son pobremente entendidos los mecanismos que gobiernan la diversidad funcional de frutos y semillas, y la efectividad de la dispersión [12], [13]. De otro lado, la selección de especies nativas asociada a semillas y otros propágulos para la restauración es un proceso complejo, ya que debe ser acorde a cada lugar, $y$ debe tener en cuenta los grupos funcionales y la sucesión vegetal, información que por lo general se 
encuentra de forma imprecisa, lo que trae consigo, fracasos de la restauración [14], [15], [13].

Una de las problemáticas frecuentemente escuchadas por parte de los restauradores, consiste en la dificultad de hacer clasificación ecológica de las especies, es decir agrupar las especies de plantas en grupos ecológicos y funcionales, esto incluye el manejo y comportamiento de las especies [16]. Por ejemplo, para Garwood [17] las clasificaciones de plántulas no son $100 \%$ precisas, pero han sido muy útiles para proveer información sobre la importancia ecológica y funcional de los rasgos morfológicos iniciales de las plantas. Aunque no existe un consenso entre los investigadores respecto a la clasificación ideal, es frecuente encontrar en la literatura clasificaciones o agrupaciones útiles y funcionales. Por ejemplo, algunos autores, clasifican especies de plantas dependiendo de su dispersión (autocoria, anemocoria, y zoocoria) [18], [19], otros lo hacen con base a los requerimientos de luz de las plantas [20], al tamaño del claro [21], incluso los términos utilizados para denominar el grupo ecológico puede variar, para Martinez- Ramos [22] las especies pueden agruparse como pioneras, nómadas y tolerantes, mientras que para Kageyama y Viana [23] las especies se agrupan entre pioneras, secundarias iniciales, secundarias tardías y clímax.

Desde el punto de vista de la restauración ecológica, una clasificación muy detallada de las especies puede ser confusa para los investigadores. Algunas propuestas simplifican y agrupan las especies en dos tipos: pioneras y no pioneras, estas últimas llamadas también persistentes [21], [24]. Esta clasificación está basada en la autoecología de las especies y su ocupación dentro de la regeneración natural del bosque [24].

En el presente trabajo se quiere aportar información general sobre frutos y semillas encontrados en el BHMB, la capacidad de germinación de algunas de estas especies y su agrupación ecológica básica (pioneras y no pioneras). Se espera que esta información sirva como herramienta para la selección y posterior uso de especies de BHMB en procesos de restauración ecológica y/o compensación ambiental.

\section{MATERIALES Y MÉTODOS}

\section{Área de estudio}

El presente estudio se realizó en un bosque alto andino de 500 ha, ubicado en la vereda La Honda al occidente de la cabecera municipal de El Carmen de Viboral, en límites con el municipio de La Unión, departamento de Antioquia. Las coordenadas son: $5^{\circ} 56.293^{\prime}$ al norte y $75^{\circ} 17.107^{\prime}$ al occidente. El área de muestreo presenta una altitud de 2,587 m.s.n.m, una temperatura promedio de $14^{\circ} \mathrm{C}$ y una precipitación anual promedio de 2,402 $\mathrm{mm}$. De acuerdo con Holdrige [25] el área se encuentra en la zona de vida Bosque muy húmedo Montano Bajo (figura, 1).

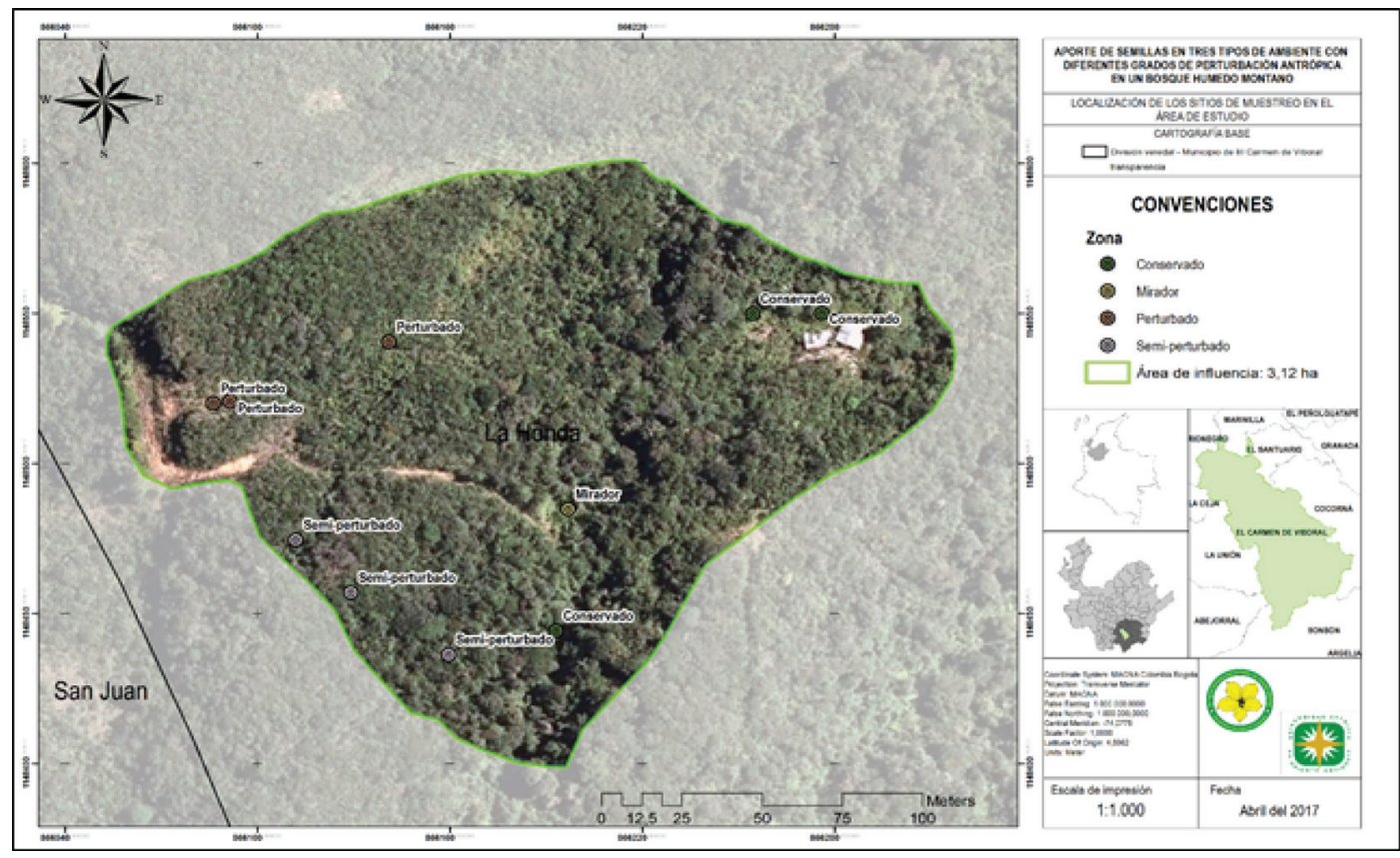

Figura 1. Ubicación geográfica del área y zonas de estudio. 
Jorge A. Sierra-Escobar - Mario Alberto Quijano Abril - Daniela Marín-Henao Daniela Salazar Suaza - Mabel Vanessa García Garcés

\section{Selección de las zonas en el área de estudio}

De acuerdo con la de intervención antrópica se seleccionaron tres clases de áreas dentro del bosque que presentaban atributos contrastantes y fácilmente diferenciables (tabla 1.). Se aclara que en cada zona fueron implementados varios transectos con fines de muestreo, los cuales no necesariamente presentaban una ubicación continua.

Tabla 1. Selección de zonas dentro del bosque para su evaluación

\begin{tabular}{cl}
\hline Clasificación del Área & \multicolumn{1}{c}{ Descripción } \\
\hline $\mathbf{1}$ & $\begin{array}{l}\text { El tipo de área } 1 \text { fue la más intervenida, presentando discontinuidad en su dosel, con } \\
\text { evidentes cambios de estructura y composición de la vegetación. Esta área estuvo constituida } \\
\text { principalmente por pastos y arbustos de poca altura fustal y parches de suelo sin ningún tipo de } \\
\text { cobertura. Además, los suelos presentaron cambios debido a actividades antrópicas anteriores } \\
\text { en el borde del bosque, principalmente por la construcción de una carretera, viviendas y áreas } \\
\text { agrícolas. Estas actividades fueron realizadas hace aproximadamente 20 años. }\end{array}$ \\
\hline $\mathbf{2}$ & $\begin{array}{l}\text { El área } 2 \text { presentó una intervención moderada, debido a que el bosque ha venido siendo } \\
\text { utilizado para la extracción de madera. Dicha extracción ha afectado la composición de } \\
\text { especies y su riqueza, en especial las maderables. La extracción de madera en este bosque } \\
\text { terminó en los años 80, debido a la presencia de grupos armados. }\end{array}$ \\
& $\begin{array}{l}\text { Elárea 3, es la más conservada del bosque remanente, debido posiblemente a las dificultades } \\
\text { de acceso, lo que evitó la extracción de madera. Es la zona con presencia de árboles de buen } \\
\text { porte, dosel cerrado, y presencia de palmas y epífitas en su sotobosque. Está zona sirvió } \\
\text { como referencia ya que su composición y estructura ha sido poco alterada. De acuerdo con } \\
\text { pobladores de la región, esta zona ha permanecido intacta en los últimos 200 años. }\end{array}$ \\
\hline
\end{tabular}

\section{Análisis de suelos}

En todas las áreas se tomaron muestras de suelo para su posterior análisis. Los análisis de las variables físicas fueron realizados en el laboratorio de suelos de la Universidad Católica de Oriente. Para dichos efectos se utilizó la metodología propuesta por Jaramillo [26] en donde se evaluó: perfil, estructura, color, densidad aparente, porcentaje de materia orgánica y $\mathrm{pH}$ de cada muestra (tabla 6). Los parámetros químicos fueron realizados por un laboratorio comercial, el cual utilizó las metodologías propuestas por Soil Survey Laboratory, en donde se analizó: pH del suelo: 1:1 (v) pH-metro; Materia Orgánica (M.O.): Walkley \& Black; Al extractable: $1 \mathrm{M} \mathrm{KCl} ; \mathrm{Ca}, \mathrm{Mg}, \mathrm{K}: 1 \mathrm{M}$ Acetato de amonio $\mathrm{pH} 7$; capacidad de intercambio catiónico efectiva (C.E.C). Fe: Olsen-EDTA. Los resultados y las metodologías para las variables químicas se encuentran en las tablas 5 y 6 .

\section{Análisis florístico}

En cada una de las zonas se realizó un inventario florístico, representado por nueve transectos de acuerdo a la metodología propuesta por [27] ISA-JAUM (2001). A cada individuo colectado al lado derecho del transecto se le midieron variables como DAP, Altura total (HT) y Fustal (HF), hábito de crecimiento de la especie y toda la información dendrológica necesaria para su identificación, como presencia de exudados, olores, entre otros [28], [29]. Al lado izquierdo solo se colectaron muestras botánicas de las especies que tuvieran un DAP mayor a 2,5 cm (figura 2).

Para el proceso de herborización (colecta, secado, identificación, e inclusión en el herbario) del material colectado, se aplicó la metodología propuesta por Lisner [30] . Todas las muestras fueron llevadas y depositadas en el herbario de la Universidad Católica de Oriente (HUCO). 


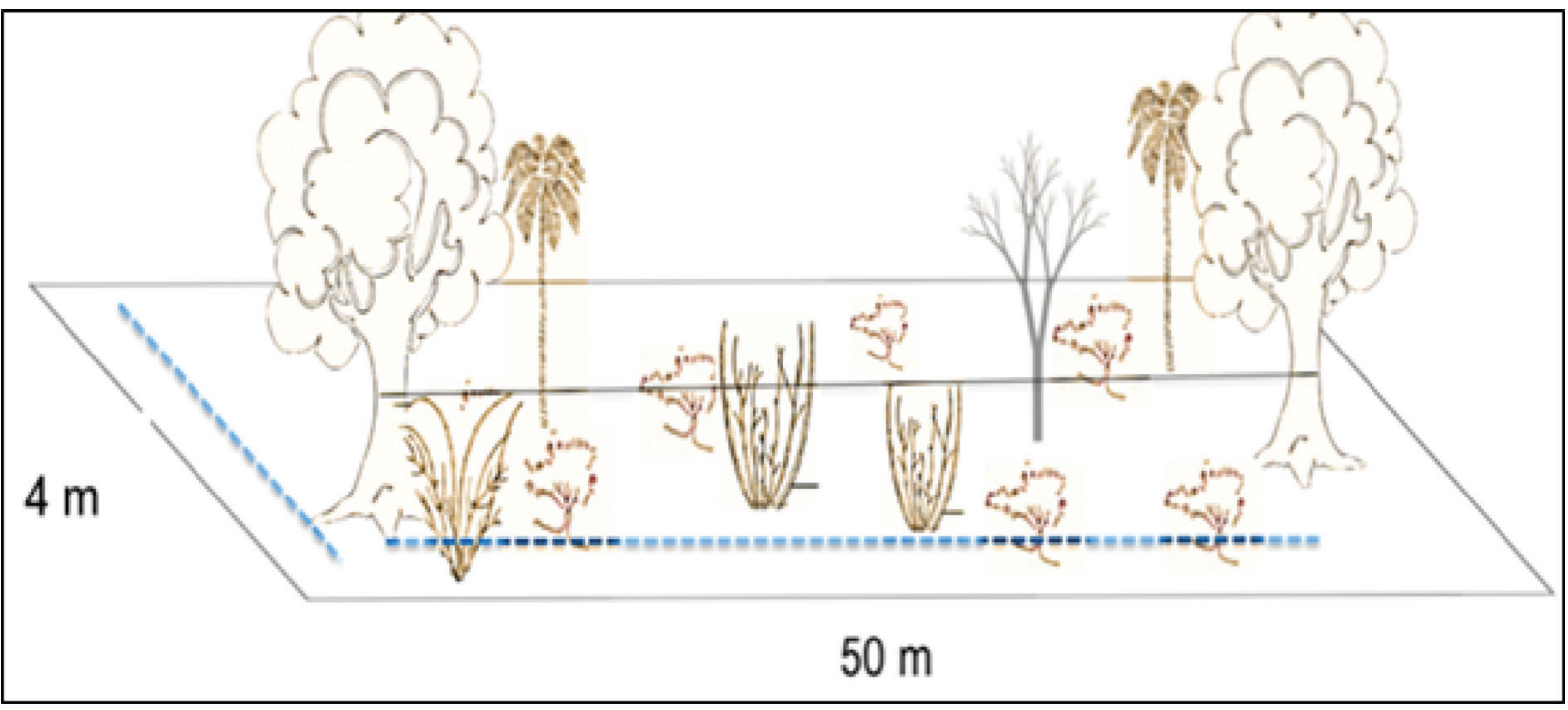

Figura 2. Esquema del censado y medición del RAP modificado ISA-JAUM 2001

Para la descripción de la estructura de la vegetación del bosque y resaltar las especies más representativas de la zona, se tuvo en cuenta el número de estratos (estructura vertical) mediante hábitos de crecimiento y perfiles esquemáticos empleando la información de uno de los transectos. Para esto se siguió la metodología propuesta por Rangel y Lozano [31] ajustada para ecosistemas andinos, en la cual se contemplan los siguientes tipos de estratos: arbustivo (1,5-5 m.); subarbóreo (5-12 m.); arbóreo inferior (12-25 m.) y arbóreo superior (25-28 m. ). La estructura horizontal del bosque se describe a partir de la distribución del número de árboles por clase diamétrica [32].

Para cuantificar la diversidad dentro del bosque se calcularon los índices de diversidad de Shannon, Simpson y Margalef. Además, con el fin de analizar el predominio de las especies en las diferentes zonas de estudio se calculó el índice de valor de importancia (IVI) de acuerdo a la metodología propuesta por Curtis and McIntosh [33].

\section{Especies pioneras y persistentes.}

Las especies de árboles y arbustos encontradas, se separaron en dos grupos ecológicos, de acuerdo a su estado sucesional (pioneras y no pioneros o persistentes), todo esto basado en la autoecología y la ocupación de cada especie dentro del bosque o su regeneración, basado en la metodología propuesta por Martínez-Ramos et al. [20]. Las especies pioneras fueron asociadas a grandes claros, áreas intervenidas, luz directa, o cercanía a cultivos y carreteras. Las especies persistentes (no pioneras) fueron asociadas a las encontradas en el interior del bosque donde se observaron también plántulas que se desarrollaban con el dosel cerrado y con bajos requerimientos de luz. A su vez, fueron incluidas aquellas que se encontraban en claros pequeños, con baja intensidad de luz solar directa.

\section{Frutos y semillas}

- Muestreo de frutos. Se realizaron visitas periódicas a todas las zonas, realizando en ellas observaciones de campo e identificando las plantas que presentaban fructificación. Una vez reconocidas las plantas fructificadas, se tomaron datos de interés para la identificación de estas, tales como: altura de la planta, DAP, número de frutos por planta, color del fruto, exudados, olores, entre otros. Toda esta información fue diligenciada en formatos previamente elaborados, para posteriormente realizar el debido registro fotográfico tanto de la planta, como del fruto y la semilla. Cada individuo fue colectado utilizando cinta adhesiva y bolsas de papel previamente marcadas con el número de colecta y lugar, para ser llevado y procesado en el Herbario de la Universidad Católica de Oriente (HUCO). Una vez en el laboratorio, se realizaron mediciones y cortes transversales de cada uno de ellos, con el objetivo de visualizar diferentes aspectos morfológicos y tipos de frutos. Los frutos fueron clasificados como secos, carnosos, dehiscentes e indehiscentes, dando así un adecuado manejo de las semillas. En el 
Jorge A. Sierra-Escobar - Mario Alberto Quijano Abril - Daniela Marín-Henao Daniela Salazar Suaza - Mabel Vanessa García Garcés

caso de los frutos dehiscentes se esperó un poco más de tiempo para que liberaran naturalmente las semillas [34].

- Conteo de semillas. Las semillas de cada fruto colectado, fueron cuidadosamente disectadas, teniendo en cuenta el número de semillas por fruto y otros aspectos morfológicos tales como: tamaño, color, textura y presencia de arilo $u$ otro tipo de exudados. Las semillas extraídas fueron lavadas para así retirar los restos de arilo o endocarpio y evitar la aparición de hongos, secándose a temperatura ambiente. Para finalizar se realizó el registro fotográfico de todas las semillas extraídas, las cuales, fueron almacenadas en bolsas plásticas herméticas con un duplicado para la colección de semillas del Herbario Universidad Católica de Oriente (HUCO) y algunas fueron seleccionadas para realizar las pruebas de germinación.

- Pruebas de germinación. Las semillas fueron secadas en el horno por 1 minuto con el fin de esterilizarlas. Algunas semillas se sembraron en medios esterilizados como papel filtro o algodón dentro de cajas de Petri. En dichos medios fueron sembradas 10 semillas por fruto y cada caja fue marcada con el respectivo código de colecta y de especie. Para dar lugar a la germinación, las semillas permanecieron a temperatura ambiente y con la luz solar indirecta durante el día. La hidratación de las mismas se realizaba cada tres días por medio de un gotero y una vez iniciado el proceso de germinación de las semillas, la fecha de germinación y el número de semillas germinadas fueron registrados en la libreta de campo e incluidos en una base de datos.

\section{Análisis de datos}

Para cuantificar la diversidad alfa dentro del bosque se calcularon los índices de diversidad de Shannon, Simpson y Margalef. Los cálculos anteriores se realizaron mediante el software Biodiversity-Pro, [35] [36]. Además, se determinó el índice de valor de importancia (IVI) [33] y se realizaron perfiles de vegetación, con el fin de hacer análisis estructurales y florísticos de cada zona.

\section{RESULTADOS}

\section{Riqueza y Composición florística del área de estudio}

En total en el bosque se encontraron 291 individuos distribuidos en 162 especies, 83 géneros y 54 familias. Datos similares encontró Ocaña-Figueroa [37] en bosques andinos de Santander, evidenciando 128 especies, 78 géneros y 41 familias. De acuerdo a los datos, el área 3 y el área 1, presentaron una mayor cantidad de especies, con valores de $75 \mathrm{y}$ 74 respectivamente, por el contrario, el área 2, presentó el número más bajo de especies, 43 en total (tabla 2).

Tabla 2. Número de familias, géneros y especies de cada zona

\begin{tabular}{cccc}
\hline Zona & Familia & Géneros & Especies \\
\hline $\mathbf{3}$ & 37 & 51 & 75 \\
$\mathbf{2}$ & 27 & 36 & 43 \\
$\mathbf{1}$ & 34 & 51 & 74 \\
\hline
\end{tabular}

Las familias más representativas encontradas para todas las áreas fueron: Aquifoliaceae (1 género y 5 especies), Asteraceae (5 géneros y 7 especies), Clusiaceae (2 géneros y 5 especies), Ericaceae (4 géneros y 8 especies), Melastomataceae (6 géneros y 16 especies) y Rubiaceae (2 géneros y 10 especies). Los géneros más frecuentes fueron:
Cavendishia (4 especies), Clusia (4 especies), Ilex (5 especies), Miconia (11 especies) y Palicourea (9 especies). Si bien las características de cobertura de copa, estructura vertical y diversidad de vegetación en las tres zonas son muy diferentes (figuras 3, 4 y 5), se encontraron cinco especies comunes entre ellas, las cuales fueron: Clethra fagifolia, Clusia 
Jorge A. Sierra-Escobar - Mario Alberto Quijano Abril - Daniela Marín-Henao Daniela Salazar Suaza - Mabel Vanessa García Garcés

multiflora, Cavendishia pubescens, Palicourea angustifolia y Vismia laevis.

De las especies encontradas durante el inventario florístico, se destacan dos nuevos registros para la ciencia en el género Schefflera (Araliaceae), las cuales se encuentran en proceso de descripción. Así mismo se han encontrado especies de palmas y orquídeas que se encuentran registradas en los libros rojos a nivel nacional como es el caso de: Ceroxylon quindiuense (EN), Bactris coloniata (LC), Euterpe precatoria (LC), Geonoma undata (NT) y Miltoniopsis vexillaria (VU) [38].

\section{Índices de riqueza y diversidad}

El bosque en general refleja alta diversidad beta, pues al comparar las tres áreas, se aprecia una marcada diferenciación entre las especies encontradas.
Como se mencionó anteriormente solo cinco especies de las 162 se encuentran en todas las áreas. En cuanto al índice de Simpson, se encontraron valores superiores a 0.9 , lo que sugiere que no existe un predominio marcado de pocas especies. El índice de Shannon también arrojó valores importantes ya que dos de los sitios evaluados, área C (Bosque conservado) y área $\mathrm{A}$ (Bosque intervenido) presentaron datos superiores a 3.3, lo que refleja una considerable diversidad. En cuanto al índice de Margalef, todas las áreas evaluadas presentaron alta diversidad con valores superiores a 2.0. Cuando los datos son inferiores a esta cantidad, pueden ser consideradas como áreas de baja riqueza específica [39]. Se aclara que el área B (Bosque medianamente intervenido) presentó valores inferiores en todos los índices con respecto a los demás sitios (tabla 3 ).

Tabla 3. Índices de Diversidad

\begin{tabular}{cccc}
\hline Índice & Conservado & $\begin{array}{c}\text { Medianamente } \\
\text { intervenido }\end{array}$ & Intervenido \\
\hline Simpson & 0.960 & 0.935 & 0.965 \\
Shannon & 3.324 & 2.804 & 3.414 \\
Margalef & 8.184 & 5.584 & 8.794 \\
\hline
\end{tabular}

En cuanto a los resultados del índice de valor de importancia, fueron seleccionadas 10 especies con valores sobresalientes, de las cuales las de mayor IVI fueron Miconia resima y Palicurea angustifolia. En general estas 10 especies, son claves para el área de estudio, debido a la alta densidad y frecuencia que presentan. Además, $P$. angustifolia, $C$. pubescens, C. multiflora, $C$. fagifolia, y $V$. laevis son comunes en las tres áreas muestreadas (tabla 4).

Tabla 4. Índice de Valor de Importancia

\begin{tabular}{llc}
\hline \multicolumn{1}{c}{ Familia } & \multicolumn{1}{c}{ Nombre científico } & IVI \\
\hline Melastomataceae & Miconia resima & 5.13 \\
Rubiaceae & Palicourea angustifolia & 4.90 \\
Rubiaceae & Palicourea garciae & 4.49 \\
Ericaceae & Cavendishia pubescens & 4.49 \\
Clusiaceae & Clusia multiflora & 4.49 \\
Clusiaceae & Clusia ducuoides & 4.07 \\
Clethraceae & Clethra fagifolia & 3.42 \\
Hypericaceae & Vismia laevis & 3.19 \\
Aquifoliaceae & Ilex danielis & 3.19 \\
Rubiaceae & Ladenbergia macrocarpa & 2.78 \\
\hline
\end{tabular}


Jorge A. Sierra-Escobar - Mario Alberto Quijano Abril - Daniela Marín-Henao Daniela Salazar Suaza - Mabel Vanessa García Garcés

\section{Características generales de cada zona.}

Suelos

Los análisis físico químicos, corroboran en parte el deterioro del suelo en el área 1 , con los valores más bajos en materia orgánica, $\mathrm{P}$, y N-N03 (tablas 5y 6), comparada con las demás, esta área presentó disminución en todos los parámetros. Por ejemplo, el pH fue de 5,4 en comparación con el $\mathrm{pH}$ de 4,3 de los otros sitios, esto es debido posiblemente al uso del suelo (tabla, 5).

Tabla 5. Parámetros químicos de las tres áreas de estudio.

\begin{tabular}{cccccccccccc}
\hline & $\mathbf{1 0}$ & \multicolumn{10}{c}{ Parámetros químicos } \\
\cline { 2 - 10 } & $\mathbf{p H}$ & & $\mathbf{N}-\mathbf{N 0 3}$ & $\mathbf{P}$ & $\mathbf{S}$ & $\mathbf{F e}$ & & $\mathbf{C u}$ & $\mathbf{Z n}$ & $\mathbf{B}$ \\
\hline $\mathbf{1}$ & 5.4 & 7.7 & 0.675 & 7 & 4 & 90 & 12 & 5 & 8 & 0.01 \\
$\mathbf{3}$ & 4.3 & 11.4 & 29 & 9 & 3 & & 1 & 21 & 1 & 0.01 \\
\hline
\end{tabular}

pH suelo: 1:1 (v.) pH-metro; Materia Orgánica (M.O.): Walkley \& Black; Al extractable: $1 \mathrm{M} \mathrm{KCl}$; $\mathrm{Ca}, \mathrm{Mg}, \mathrm{K}: 1 \mathrm{M}$ Acetato de amonio $\mathrm{pH}$ 7; capacidad de intercambio catiónico efectiva (C.E.C). Fe: Olsen-EDTA.
Como se esperaba, el área 1 presento la mayor densidad aparente, este valor fue bajando a medida que disminuía la intervención (tabla 6).

Tabla 6. Parámetros físicos de las tres zonas de estudio.

\begin{tabular}{ccccccc}
\hline & \multicolumn{5}{c}{ Parámetros físicos } \\
\cline { 2 - 7 } Zona & \% Arena & \% Arcillas & \% Limo & Textura & $\begin{array}{c}\text { \% Sat. } \\
\text { Humedad }\end{array}$ & Den. aparente \\
\hline 1 & 50 & 30 & 20 & Franco & 90.352 & 1.0 \\
2 & 74 & 14 & 12 & $\begin{array}{c}\text { Franco } \\
\text { arenosa }\end{array}$ & 122.99 & 0.9 \\
3 & 72 & 14 & 14 & $\begin{array}{c}\text { Franco } \\
\text { arenosa }\end{array}$ & 177.39 & 0.8 \\
\hline
\end{tabular}

\section{COMPOSICIÓN FLORÍSTICA}

\section{Área 1}

En esta área fue frecuente encontrar suelos desnudos, lo que favorece la erosión del suelo y cambios bruscos de temperatura. La flora documentada tuvo un predominio de arvenses y arbustos (figura 3) que fue clasificada en estrato herbáceo, arbustivo y subarbóreo. A continuación, se presentan las especies en cada estrato.

- Estrato herbáceo: Ageratina popayanensis, Ageratum conyzoides, Anthurium angosturense, Anthurium cupreum, Baccharis brachylaenoides, Burmeistera glabrata, Cavendishia Sp2, Cavendishia Sp1,
Chrysochlamys colombiana, Clethra fagifolia, Clusia ducuoides,, Clusia multiflora, Columnea consanguinea, Elleanthus aurantiacus, Emilia sonchifolia, Erato vulcanica, Guzmania multiflora, Hesperomeles obtusifolia, Ilex danielis, Lonicera japónica, Mezobromelia capituligera, Miconia jahnii, Miconia Sp, Monochaetum multiflorum, Myrsine coriácea, Nastus chusque, Palicourea angustifolia, Palicourea aschersonianoides, Palicourea demissa, Palicourea garciae, Piper artanthe, Psammisia breviflora, Rubus bogotensis, Rubus guyanensis y Vismia laevis.

- Estrato arbustivo: Ageratina popayanensis, Baccharis latifolia, Bejaria aestuans, Billia 
Jorge A. Sierra-Escobar - Mario Alberto Quijano Abril - Daniela Marín-Henao Daniela Salazar Suaza - Mabel Vanessa García Garcés

rosea, Cavendishia angustifolia, Cavendishia pubescens, Clethra fagifolia, Cyathea caracasana, Cybianthus iteoides, Disterigma acuminatum, Drimys granadensis, Graffenrieda emarginata, Hedyosmum bonplandianum, Hieronyma antioquensis, Hypericum juniperinum, Ilex danielis, Ilex laurina, Ilex pernervata, Ladenbergia macrocarpa, Miconia affinis, Miconia resima, Miconia theaezans, Monnina fastigiata, Morella pubescens, Myrsine coriácea, Myrsine pellucida, Oreopanax bogotensis, Palicourea demissa, Palicourea garciae.

- Estrato subarboreo: Aegiphila alba, Axinaea macrophylla, Brunellia goudotii, Clusia alata, Clusia ducuoides, Escallonia paniculata, Ilex kunthiana, Palicourea apicata, Vismia laevis.

Figura 3. Perfil de vegetación, área 1

8

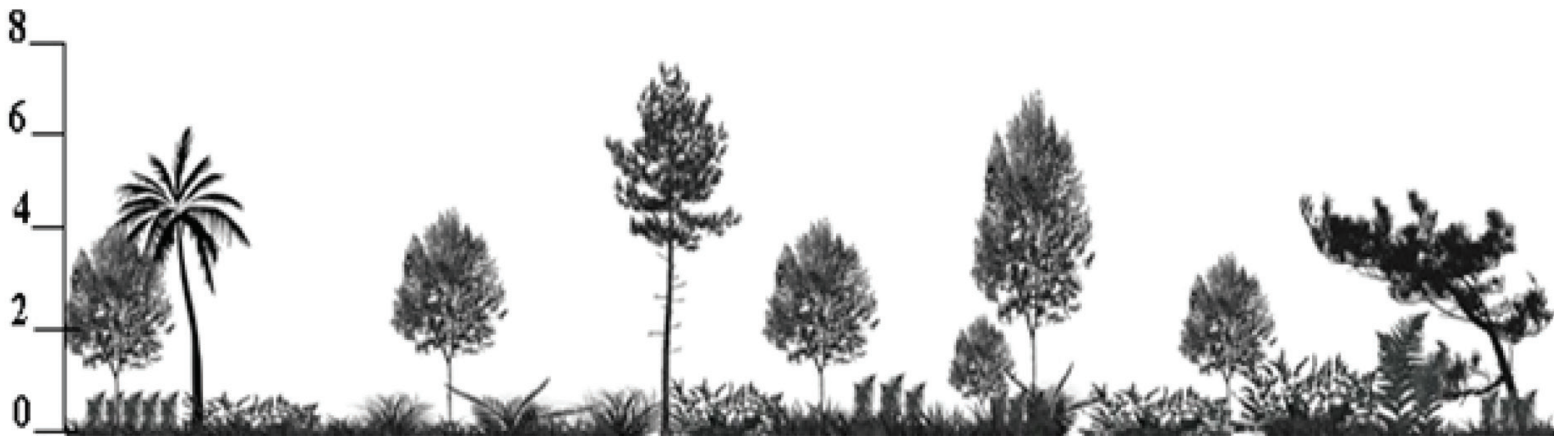

\section{Área 2}

Esta área se encuentra moderadamente intervenida y es frecuente encontrar grupos de árboles conformando un dosel, al igual que claros de tamaño variable (figura 4). Entre las especies presentes en este sitio, se destacan por su abundancia: Axinaea macrophylla, Cavendishia pubescens y Vismia laevis. No hay presencia de estrato herbáceo y las especies presentes en el estrato arbustivo y el estrato subarbóreo son mostradas a continuación.

- Estrato arbustivo: Aegiphila alba, Axinaea macrophylla, Baccharis brachyphylla, Bactris coloniata, Casearia quinduensis, Cavendishia pubescens, Clethra fagifolia, Clusia alata, Clusia ducuoides, Clusia multiflora, Drimys granadensis, Euterpe precatoria, Gaiadendron punctatum, Graffenrieda emarginata, Hedyosmum goudotianum, Ilex danielis, Ilex laurina, Meriania nobilis, Miconia wurdackii, Myrcia tomentosa, Ocotea kunthiana, Palicourea angustifolia, Palicourea demissa, Schefflera ferruginea, Tibouchina lepidota, Varronia cylindrostachya $\mathrm{y}$ Viburnum antioquiense.

- Estrato subarbóreo: Axinaea macrophylla, Billia rosea, Clusia ducu, Cybianthus marginatus, Geonoma undata, Graffenrieda emarginata, Hedyosmum goudotianum, Hieronyma huilensis, Myrcia tomentosa, Myrsine coriacea, Palicourea angustifolia, Palicourea garciae.

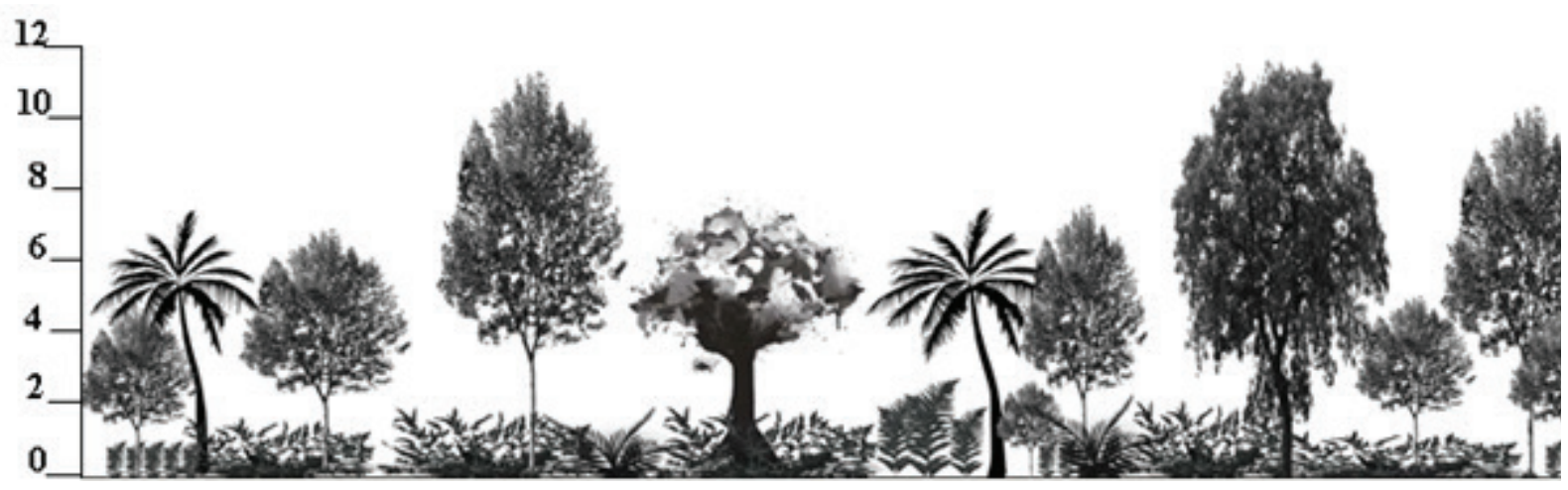

Figura 4. Perfil de vegetación, área 2 
Jorge A. Sierra-Escobar - Mario Alberto Quijano Abril - Daniela Marín-Henao Daniela Salazar Suaza - Mabel Vanessa García Garcés

\section{Área 3}

Esta área es la mejor conservada y su perfil vertical está compuesto por cuatro estratos: estrato arbustivo, estrato subarbóreo, estrato arbóreo superior y estrato arbóreo inferior (figura 5). Se destacan por su abundancia las especies: Palicourea angustifolia, Miconia resima, Clethra fagifolia y Alchornea latifolia. A continuación, se muestran las especies presentes en cada estrato.

- Estrato arbustivo: Alchornea latifolia, Aniba riparia, Annona quinduensis Kunth, Billia rosea, Cavendishia pubescens, Clethra fagifolia, Cyathea andina, Cyathea caracasana var. Meridensis, Elleanthus $S p$, Hedyosmum bonplandianum, Ilex danielis, Lacepedea pinnata, Ladenbergia macrocarpa, Meriania nobilis, Miconia asperrima, Miconia lehmannii, Miconia ligustrina, Miconia resima. Miconia resima, Monnina aestuans, Morella parvifolia, Myrcia fallax, Myrsine coriácea, Palicourea acetosoides, Palicourea angustifolia, Palicourea garciae, Palicourea guianensis, Palicourea thyrsiflora, Piper calceolarium, Saurauia chiliantha, Schefflera quinduensis, Symplocos phaeoneura, Tapura colombiana, Tontelea attenuata, Varronia cylindrostachya.
- Estrato subarbóreo: Alchornea latifolia, Alchornea verticilata, Aniba riparia, Annona quinduensis, Axinaea macrophylla, Billia rosea, Cavendishia pubescens, Chamaedorea liebmannii, Clethra fagifolia, Clusia alata, Clusia ducuoides, Clusia multiflora, Cyathea divergens, Hieronyma antioquensis, Ilex danielis, Ilex laurina, Ilex pustulosa, Lacepedea pinnata, Meriania nobilis, Miconia dolichopoda, Miconia ligustrina, Miconia resima, Morella parvifolia, Myrcia tomentosa, Nectandra lineata, Palicourea angustifolia, Palicourea garciae, Piper archeri, Piper calceolarium, Sapium stylare, Schefflera trianae, Viburnum anabaptista, Viburnum undulatum, Vismia baccifera, Vismia laevis, Weinmannia pubescens, y Zanthoxylum melanostictum.

- Estrato arbóreo inferior: Alchornea latifolia, Alchornea verticilata, Ceroxylon quindiuense, Chamaedorea liebmannii, Conceveiba parvifolia, Graffenrieda emarginata, Hieronyma antioquensis y Myrcia tomentosa.

- Estrato arbóreo superior: Conceveiba parvifolia.

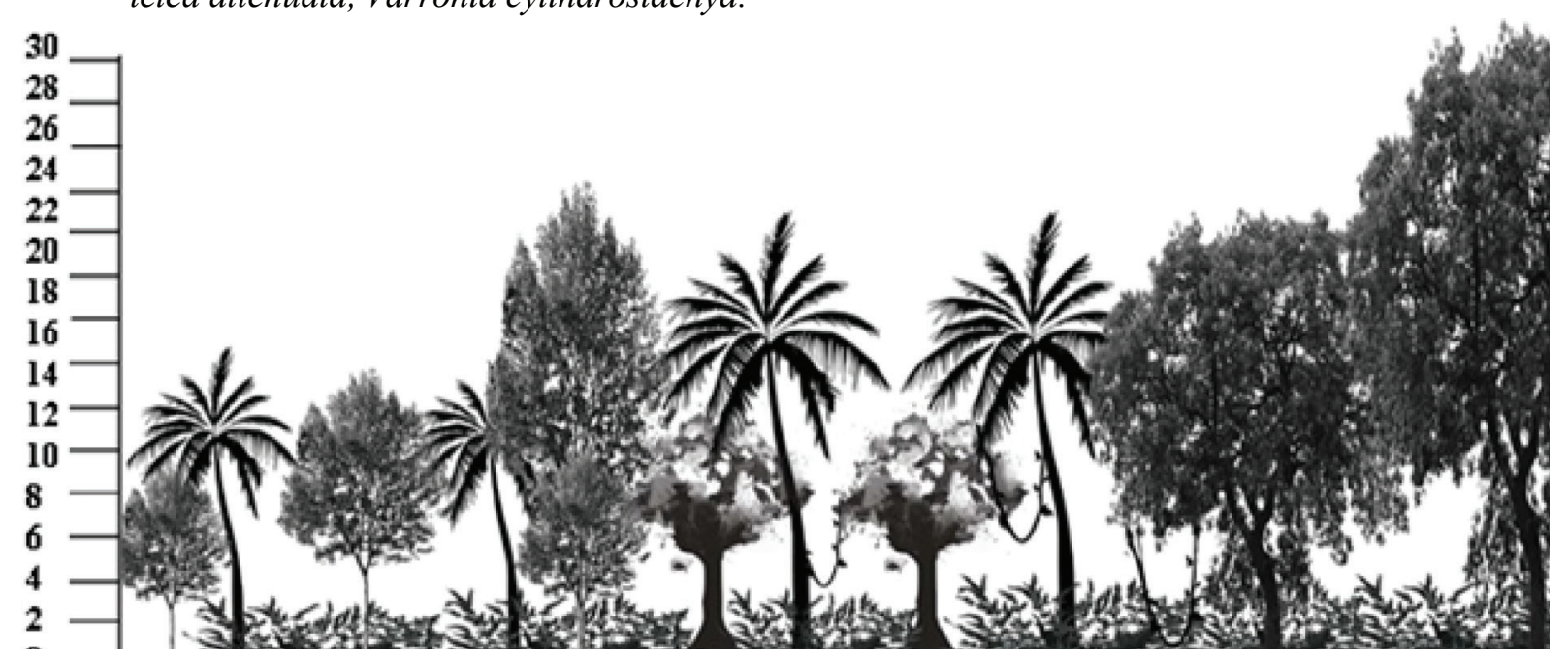

Figura 5. Perfil de vegetación, área 3.

\section{Especies pioneras y persistentes encontradas}

En restauración ecológica, es usual focalizar un ecosistema de referencia, en este caso, un bosque para entender de este la dinámica arbórea, la composición, función, y estructura [40]. Esa información es generalmente utilizada para la selección de especies con potencial para la res- tauración. Por lo general los restauradores utilizan especies pioneras en sus proyectos, ya que estás crecen rápidamente, son heliófilas, y se adaptan más fácilmente a condiciones adversas. En este estudio, se clasificaron 42 especies de árboles en dos grupos, pioneras y persistentes (no pioneras) (tabla 7). 
Jorge A. Sierra-Escobar - Mario Alberto Quijano Abril - Daniela Marín-Henao Daniela Salazar Suaza - Mabel Vanessa García Garcés

Tabla 7. Árboles pioneros y persistentes encontrados

\begin{tabular}{|c|c|c|}
\hline Nombre Científico & Familia & $\begin{array}{r}\text { Grupo } \\
\text { ecológico } \\
\end{array}$ \\
\hline Aegiphila alba Moldenke & Lamiaceae & Pionera \\
\hline Alchornea latifolia Sw. & Euphorbiaceae & Persistente \\
\hline Alchornea verticilata Renteria y Franco & Euphorbiaceae & Persistente \\
\hline Annona quinduensis Kunth & Annonaceae & Persistente \\
\hline Axinaea macrophylla (Naudin) Triana & Melastomataceae & Persistente \\
\hline Billia rosea (Planch. \& Linden) C. Ulloa \& P. Jørg. & Sapindaceae & Persistente \\
\hline Brunellia goudotii Tul. & Brunelliaceae & Pionera \\
\hline Ceroxylon quindiuense (H. Karst.) H. Wendl. & Arecaceae & Persistente \\
\hline Chamaedorea liebmannii Mart. & Arecaceae & Persistente \\
\hline Clethra fagifolia Kunth & Clethraceae & Persistente \\
\hline Clusia alata Planch. \& Triana & Clusiaceae & Pionera \\
\hline Clusia multiflora Kunth & Clusiaceae & Pionera \\
\hline Conceveiba parvifolia $\mathrm{McPherson}$ & Euphorbiaceae & Persistente \\
\hline Cyathea andina (H. Karst.) Domin & Cyatheaceae & Persistente \\
\hline Cyathea caracasana var. meridensis (H. Karst.) R.M. Tryon & Cyatheaceae & Persistente \\
\hline Cyathea divergens Kunze & Cyatheaceae & Persistente \\
\hline Cybianthus marginatus (Benth.) Pipoly & Primulaceae & Pionera \\
\hline Graffenrieda emarginata (Ruiz \& Pav.) Triana & Melastomataceae & Persistente \\
\hline Hieronyma antioquensis Cuatrec. & Phyllanthaceae & Persistente \\
\hline Hieronyma huilensis Cuatrec. & Euphorbiaceae & Persistente \\
\hline Ilex pustulosa Triana & Aquifoliaceae & Persistente \\
\hline Ladenbergia macrocarpa (Vahl) Klotzsch & Rubiaceae & Pionera \\
\hline Meriania nobilis Triana & Melastomataceae & Pionera \\
\hline Miconia lehmannii $\operatorname{Cogn}$ & Melastomataceae & Pionera \\
\hline Myrcia tomentosa (Aubl.) DC. & Myrtaceae & Persistente \\
\hline Myrsine coriacea (Sw.) R. Br. ex Roem. \& Schult. & Primulaceae & Pionera \\
\hline Nectandra lineata (Kunth) Rohwer & Lauraceae & Persistente \\
\hline Palicourea acetosoides Wernham & Rubiaceae & Persistente \\
\hline Palicourea angustifolia Kunth & Rubiaceae & Pionera \\
\hline Piper calceolarium C. DC. & Piperaceae & Persistente \\
\hline Prunus integrifolia (C. Presl) Walp. & Rosaceae & Persistente \\
\hline Sapium stylare Müll. Arg. & Euphorbiaceae & Persistente \\
\hline Schefflera ferruginea (Willd. ex Schult.) Harms & Araliaceae & Pionera \\
\hline Schefflera trianae (Planch. \& Linden ex Marchal) Harms & Araliaceae & Pionera \\
\hline Solanum nutans Ruiz \& Pav. & Solanaceae & Pionera \\
\hline Symplocos phaeoneura B. Ståhl & Symplocaceae & Persistente \\
\hline Tontelea attenuata Miers & Celastraceae & Persistente \\
\hline Varronia cylindrostachya Ruiz \& Pav. & Boraginaceae & Persistente \\
\hline Vismia baccifera (L.) Triana \& Planch. & Hypericaceae & Pionera \\
\hline Vismia laevis Triana \& Planch. & Hypericaceae & Pionera \\
\hline Zanthoxylum melanostictum Schltdl. \& Cham. & Rutaceae & Persistente \\
\hline Zanthoxylum monophyllum (Lam.) P. Wilson & Rutaceae & Persistente \\
\hline
\end{tabular}


Como se puede observar en la tabla 7, en las áreas 1 y 2 hubo un predominio de especies pioneras, esto se debe principalmente a la aparición de claros de tamaño variable, favoreciendo a este grupo ecológico. En estas especies la floración y fructificación fueron constantes a lo largo de los muestreos realizados [41]

\section{Frutos y semillas}

Se colectaron aproximadamente 114 frutos en las tres áreas muestreadas, de los cuales a 100 frutos fue posible extraerles las sus semillas para pruebas de germinación. Cuarenta especies presentaron germinación con valores que oscilan entre el 10 y el $100 \%$, mientras que en las semillas de 60 especies la germinación fue nula (tabla 8). La baja germinación o la ausencia de esta se debió en su mayoría a la contaminación de las semillas en el proceso de almacenamiento y en las cajas de Petri, ya que a pesar de que se realizó esterilización de los medios y de las semillas se observó en repetidas ocasiones la presencia de hongos. En el área 1 y 2 se encontró un mayor número de frutos y semillas, posiblemente debido al predomino de arvenses y arbustos, entre los cuales las arvenses presentaron porcentajes de germinación por encima del $90 \%$. En la tabla 8 solo se presentan datos principalmente de árboles y arbustos, debido a que estos son más frecuentemente usados en procesos de restauración ecológica.

Tabla 8. Generalidades de algunas especies estudiadas.

\begin{tabular}{|c|c|c|c|c|c|c|}
\hline Especie & $\begin{array}{c}\text { Habito } \\
\text { Crecimiento }\end{array}$ & $\begin{array}{c}\text { Tamaño } \\
\text { fruto }(\mathbf{c m}) \\
\text { Largo/ } \\
\text { Ancho }\end{array}$ & $\begin{array}{c}\text { Semillas } \\
\text { por } \\
\text { fruto }\end{array}$ & $\begin{array}{c}\text { Tamaño } \\
\text { semilla } \\
(\mathbf{m m}) \\
\text { Largo/ } \\
\text { Ancho }\end{array}$ & $\begin{array}{c}\text { Germinación } \\
(\%)\end{array}$ & $\begin{array}{c}\text { Zona } \\
\text { colecta }\end{array}$ \\
\hline Coccocypselum hirsutum & Hierba & $1.7 / 0.7$ & 8 & $1.5 / 1.0$ & 90 & $\mathrm{Z1}$ \\
\hline Galium hypocarpium & Hierba & $0.4 / 0.5$ & 2 & $2.0 / 1.5$ & 60 & $\mathrm{Z1}$ \\
\hline Morella pubescens & Arbusto & $0.5 / 0.5$ & 1 & $3.0 / 2.5$ & 70 & $\mathrm{Z1}$ \\
\hline Miconia myrtillifolia & Arbusto & $0.9 / 1.0$ & Más de 1000 & $0.5 / 0.25$ & 90 & $\mathrm{Z1}$ \\
\hline Cavendishia guatapeensis & Arbusto & $0.5 / 1.0$ & $\begin{array}{c}\text { Entre } \\
200-300\end{array}$ & $1.0 / 0.5$ & 0 & $\mathrm{Z1}$ \\
\hline Vismia laevis & Arbusto & $1.6 / 0.9$ & 35 & $1.5 / 1.0$ & 0 & $\mathrm{Z1}$ \\
\hline Macrocarpea macrophylla & Arbusto & $2.4 / 0.7$ & $\begin{array}{c}\text { Más de } \\
1,000\end{array}$ & $0.6 / 0.5$ & 10 & $\mathrm{Z1}$ \\
\hline Monnina solandrifolia & Arbusto & $0.5 / 0.3$ & 1 & $5.0 / 2.1$ & 0 & $\mathrm{Z1}$ \\
\hline Cladocolea archeri & Parásita & $0.5 / 0.4$ & 1 & $5.0 / 3.0$ & 0 & $\mathrm{Z1}$ \\
\hline Shefflera sp & Árbol & $0.6 / 0.4$ & 3 & $3.0 / 1.2$ & 0 & $\mathrm{Z1}$ \\
\hline Centropogum sp. & Hierba & $2.6 / 3.5$ & 250 A 300 & $1.0 / 0.5$ & 100 & $\mathrm{Z1}$ \\
\hline Blakea albertiae & Árbol & $0.9 / 1.0$ & 200 & $1.2 / 1.0$ & 0 & $\mathrm{Z1}$ \\
\hline Miconia resima & Árbol & $0.4 / 0.2$ & 200 & $0.1 / 0.2$ & 0 & $\mathrm{Z1}$ \\
\hline Cybianthus iteoides & Arbusto & $0.6 / 0.3$ & 1 & $1.0 / 1.0$ & 0 & $\mathrm{Z1}$ \\
\hline Palicourea apicata & Arbusto & $0.7 / 0.6$ & 2 & $5.0 / 4.5$ & 0 & $\mathrm{Z1}$ \\
\hline Dendrophtora linteniana & Trepadora & $0.5 / 0.4$ & 1 & $2.0 / 1.0$ & 0 & $\mathrm{Z1}$ \\
\hline Varronia cylindrostachya & Arbusto & $0.5 / 0.5$ & 1 & $3.0 / 3.0$ & 0 & $\mathrm{Z1}$ \\
\hline Nertera granadensis & Hierba Rastrera & $0.6 / 0.6$ & 2 & $3.0 / 2.0$ & 0 & $\mathrm{Z1}$ \\
\hline
\end{tabular}


Jorge A. Sierra-Escobar - Mario Alberto Quijano Abril - Daniela Marín-Henao Daniela Salazar Suaza - Mabel Vanessa García Garcés

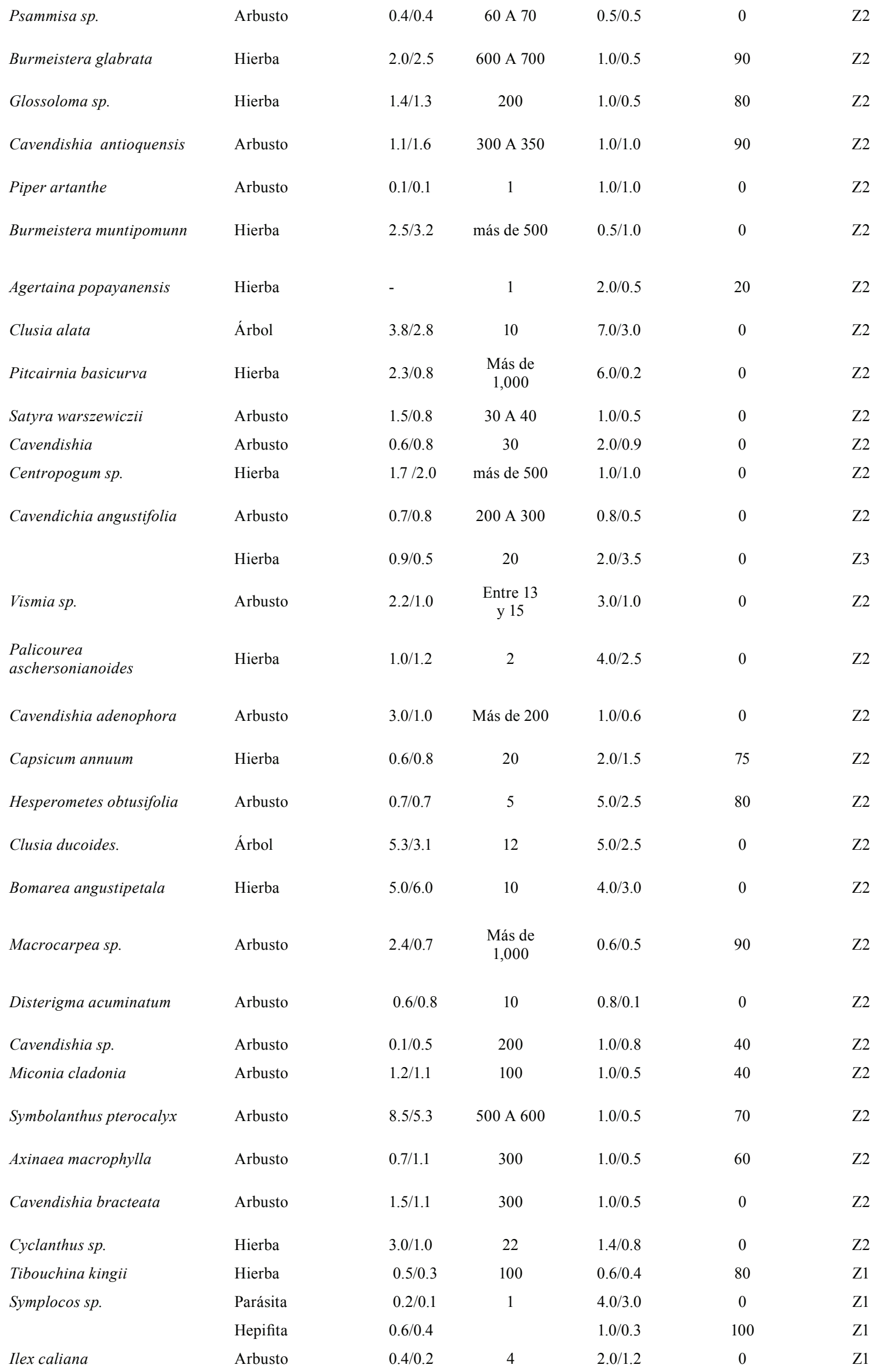


Jorge A. Sierra-Escobar - Mario Alberto Quijano Abril - Daniela Marín-Henao Daniela Salazar Suaza - Mabel Vanessa García Garcés

\begin{tabular}{|c|c|c|c|c|c|c|}
\hline Viburnum & Arbusto & $0.6 / 0.6$ & 1 & $1.3 / 4.5$ & 0 & $\mathrm{Z1}$ \\
\hline Burmeistera motipomum & Arbusto & $5.2 / 2.3$ & más de 500 & $0.8 / 0.1$ & 20 & $\mathrm{Z} 1$ \\
\hline Guzmania conifera & Hepífita & $4.2 / 0.5$ & más de 500 & $0.2 / 0.1$ & 0 & $\mathrm{Z} 2$ \\
\hline Blakea calyptrata & Arbusto & $1.1 / 1.0$ & más de 500 & $1.1 / 0.8$ & 30 & $\mathrm{Z} 2$ \\
\hline Palicurea sp. & Arbusto & $0.4 / 0.2$ & 60 & $5.0 / 3.1$ & 0 & $\mathrm{Z} 2$ \\
\hline Solanum oblongifolium & Arbusto & $1.5 / 1.2$ & Más de 200 & $3.1 / 2.8$ & 100 & $\mathrm{Z} 2$ \\
\hline Vaccinium meridionale & Arbusto & $6.5 / 7.2$ & Más de 500 & $1.5 / 1.0$ & 10 & $\mathrm{Z} 2$ \\
\hline Varronia cylindrostachya & Arbusto & $0.5 / 0.4$ & 1 & $7.0 / 4.0$ & 0 & $\mathrm{Z} 2$ \\
\hline Saurauia chiliantha & Arbusto & $1.5 / 1.4$ & & $1.3 / 1.0$ & 20 & $\mathrm{Z} 2$ \\
\hline Miconia legmanii & Arbusto & $0.4 / 0.5$ & Más de 300 & $6.0 / 0.5$ & 10 & $\mathrm{Z} 2$ \\
\hline Gaiandendron emarginata & Arbusto & $1.4 / 1.1$ & 1 & $5.0 / 3.0$ & 0 & $\mathrm{Z} 2$ \\
\hline Ilex laurina & Arbusto & $0.5 / 0.6$ & 4 & $4.0 / 1.5$ & 0 & $\mathrm{Z} 2$ \\
\hline Racinaea penlandii & Hepifita & $1.4 / 0.3$ & 10 & $2.0 / 0.1$ & 0 & $\mathrm{Z} 2$ \\
\hline Meriania nobilis & Arbusto & $2.0 / 1.8$ & más de 500 & $2.0 / 0.7$ & 60 & $\mathrm{Z} 2$ \\
\hline Cavendishia pubescens & Arbusto & $0.8 / 0.8$ & 150 & $1.0 / 0.1$ & 10 & $\mathrm{Z} 2$ \\
\hline Meriania nobilis & Arbusto & $2.0 / 1.8$ & 300 & $2.0 / 1.0$ & 20 & $\mathrm{Z} 1$ \\
\hline Disterigma alaternoides & Arbusto & $0.1 / 0.1$ & 100 & $2.0 / 1.0$ & 10 & $\mathrm{Z1}$ \\
\hline
\end{tabular}

\section{DISCUSIÓN}

Conocer la flora de los bosques cercanos a las áreas a restaurar, es muy importante, ya que en muchos casos es de las pocas opciones que se tienen para conocer cuales especies estaban presentes en el área afectada. De acuerdo con Vargas et al. [40], definir un ecosistema de referencia no es sencillo, ya que depende de las descripciones ecológicas y listados de especies antes de la perturbación. La información florística aquí obtenida, esta corroborada por otros estudios en bosques andinos [37], [42], [43]. Asimismo, de acuerdo a Barrera et al. [44] la caracterización de la vegetación vascular, permite el desarrollo de estrategias de restauración, lo que finalmente contribuirá al equilibrio de los ecosistemas disturbados.

Desde el punto de vista de la composición, estudios realizados en los cerros orientales de Bogotá, arrojaron promedios de 3.61 para el índice de Shannon y 0.97 para el índice de Simpson [45], lo que concuerda con los resultados aquí obtenidos (tabla 3). Esto es posiblemente debido a que ambos estudios se realizaron en condiciones ambientales similares.
Es interesante anotar que C. multiflora, y otras especies del género Vismia, han sido reportadas con valores de IVI altos en bosques andinos de Santander [37]. Otros autores han sugerido el uso de individuos del género Vismia para revegetalizar áreas degradadas en combinación con algunas arvenses [46]. Además, especies de las familias Rubiaceae, Melastomataceae, Lauraceae, Arecaceae y Euphorbiaceae, fueron encontradas con frecuencia en las áreas 1 y 2, algunas en áreas con claros grandes, dinámica ya reportada por Rincón-Velázquez et al. [43].

El conocimiento de la sucesión vegetal y los grupos ecológicos a partir de los ecosistemas de referencia [40], han facilitado el trabajo de los restauradores. Algunos autores lo han denominado el enfoque sucesional, porque permite predecir la dinámica de las especies en el corto y largo plazo, lo que facilita sugerir las futuras trayectorias del modelo propuesto [47]. Un método frecuentemente utilizado consiste en establecer un grupo pequeño de especies pioneras (como las que se presentan en la tabla 7) que produzcan rápidamente una cubierta de copas. En este modelo, la sombra se 
encarga de controlar malezas y pastos, y permite la colonización de nuevas especies procedentes de bosques cercanos [48]. Pero no solo las especies pioneras son adecuadas para la restauración, mezclas de diferentes grupos ecológicos han sido utilizadas con éxito [49], incluso en Brasil han incursionado implementando el enfoque fitosociológico para la restauración, que incluye a todos los grupos ecológicos con excelentes resultados [47]. Se debe tener en cuenta que la selección de especies debe ser acorde a las particularidades de cada caso, porque dependen del estado del área a restaurar (factores bióticos y abióticos), historia de usos del suelo, resiliencia del ecosistema, y el contexto del paisaje [50]. No tener identificados los disturbios, y una definición clara de lo que se quiere alcanzar en restauración genera errores que pueden afectar negativamente las trayectorias deseadas. Por ejemplo, en Sao Pablo Brasil se presentó un caso emblemático de fracaso al seleccionar las especies. En esta experiencia los investigadores utilizaron especies pioneras en áreas muy degradadas, las cuales estaba aisladas de bosques nativos. Este aislamiento generó una sucesión arrestada debido a que no llegaban propágulos del exterior a los sitios y finalmente la mayoría de las especies murieron [47]. En este caso, los investigadores omitieron el aislamiento de las áreas y por eso la selección de especies no fue la adecuada. En conclusión, la definición de especies debe ser una decisión de los restauradores, que será basada en las condiciones del sitio, y el conocimiento que se tenga sobre el estado del ecosistema que se va a restaurar.

Pero no basta con seleccionar bien las especies para la restauración, también es importante conocer de manera integral el comportamiento y manejo de las especies nativas que serán utilizadas en el ecosistema, además, obtener de forma masiva propágulos de especies nativas, no es tarea fácil [51]. A partir de fuentes semilleras procedentes de ecosistemas de referencia, se puede adquirir información que ayude suplir las necesidades que requiera el restaurador [52] y también se facilita la iniciación de estudios de ecología básica de semillas como los aquí propuestos, que incluyen porcentajes de germinación, tamaños de frutos y semillas, y cantidad de semillas producidas. Existe una experiencia muy interesante en la mata atlántica brasilera, en donde se quiere restaurar 15 millones de hectáreas de bosque tropical en 40 años, su estrategia se basa en tecnología efectiva que se somete a mejoras permanentes, enseñanza continua, esfuerzos de divulgación y desarrollo de capacidades, presencia de intelectuales locales, mantenimiento de un entorno legal claro y transparente y presencia de instrumentos económicos e incentivos efectivos para los propietarios de tierras. En esta iniciativa, cada comunidad maneja los ecosistemas de referencia, y en compañía de los investigadores seleccionan y manejan las especies que se utilizaran de forma específica en cada área a restaurar [53].

\section{CONCLUSIONES}

De acuerdo con las características generales del área de estudio, el bosque estudiado sería un buen ecosistema de referencia para futuros proyectos de restauración ecológica en el oriente antioqueño. Debido a que cuenta con una buena disponibilidad de propágulos tanto de árboles como de arbustos pioneros y persistentes, lo cual se traduce en materia prima para dotar viveros de conservación, y a su vez, se garantizaría la producción masiva de plántulas acordes a las necesidades del restaurador.

Trabajos como este muestran que es posible pensar en una propagación exitosa de especies silvestres en este tipo de ecosistemas. Es necesario generar protocolos de propagación de especies nativas en estos bosques con miras a la selección de especies, que contribuyan en procesos de restauración ecológica y no simplemente de reforestación.

\section{AGRADECIMIENTOS}

Agradecemos a la Dirección de Investigación y Desarrollo de la Universidad Católica de Oriente, por la financiación del presente proyecto. Además, a todos los estudiantes del grupo de Estudios Florísticos que ayudaron en todas las fases del proyecto.

\section{REFERENCIAS}

[1] L. S. Hamilton, "Una Campaña por los bosques nublados: Ecosistemas únicos y valiosos en peligro", de Bosques Nublados del Neotrópico, Costa Rica, INBio, 2001, p. 41 - 45.

[2] A. Etter y W. Van Wyngaarden, "Patterns of Landscape Transformation in Colombia, 
Jorge A. Sierra-Escobar - Mario Alberto Quijano Abril - Daniela Marín-Henao Daniela Salazar Suaza - Mabel Vanessa García Garcés

with Emphasis in the Andean Region", Ambio, vol. 29, n 7, pp. 443-450, 2000.

[3] K. Oyama y A. Castillo, Manejo, conservación y restauración de recursos naturales en México: perspectivas desde la investigación científica, México: UNAM / Siglo XXI Editores, 2005.

[4] P. Velasco-Linares y O. Vargas-Ríos, "Problemática de los bosques altoandinos", de Estrategias para la restauración ecológica del bosuqe alto andino: El caso de la Reserva Forestal Municipal de Cogua, Bogotá, Grupo de Restauración Ecológica, Universidad Nacional de Colombia, 2008, pp. 41-56.

[5] W. Ramírez, C. Murcia, M. R. Guariguata, E. Thomas, M. Aguilar y P. Isaac, "Restauración Ecológica", de Biodiversidad 2015. Estado y Tendencias de la Biodiversidad Continental de Colombia, Bogotá, Instituto de Investigación de Recursos Biológicos Alexander von Humboldt, 2016.

[6] UICN, El Desafío de Bonn: Catalizando liderazgo en América Latina, Forest Brief, 2007.

[7] O. Vargas, "Restauración ecológica: Biodiversidad y Conservación", Acta Biológica Colombiana, vol. 16, n 2, pp. 221-246, 2011.

[8] C. Murcia y M. R. Guariguata, La restauración ecológica en Colombia: Tendencias, necesidades y oportunidades, Bogor: Centro para la Investigación Forestal Internacional (CIFOR), 2014.

[9] G. Williams-Linera, M. Bonilla y F. LópezBarrera, "Tropical cloud forest recovery: the role of seed Banks in pastures dominated by an exotic grass", New Forests, vol. 47, n 3 , 2016.

[10] X. García y M. Martinéz-Ramos, "Sucesión ecológica y restauración de las selvas húmedas", Boletín de la Sociedad Botánica de México, vol. 80, no 080, pp. 69-84, 2006.

[11] J. L. Reid, K. D. Holl y R. A. Zahawi, "Seed dispersal limitations shift over time in tropical forest restoration", Ecological Applications, vol. 25, $\mathrm{n}^{\circ}$ 4, p. 10, 2015.

[12] H. M. Chapman, N. J. Cordeiro y P. E. Dutton, "Seed-dispersal ecology of tropical montane forests", Journal of Tropical Ecology, vol. 1, pp. 1-18, 2016.
[13] L. Yang, R. Sailesh, R. D. Harrison, X. Jianchu, O. Xiaokun, M. Xuelan y H. Jun, "Selection of Native Tree Species for Subtropical Forest Restoration in Southwest China", PLOS ONE, 2017.

[14] "The Ecology and Ecosystem Services of Native Trees: Implications for Reforestation and Land Restoration in Mesoamerica", Forest Ecology and Management, vol. 261, $\mathrm{n}^{\mathrm{o}} 10,2011$.

[15] S. Cao, L. Chen, D. Shankman, C. Wang, X. Wang y H. Zhang, "Excessive reliance on afforestation in China's arid and semi-arid regions: Lessons in ecological restoration", Earth-Science Reviews, vol. 104, no 4, pp. 240-245, 2011.

[16] M. Aguilar-Garavito, J. P. Pinzón, J. Sierra, J. Rubio y M. Quijano Abril, La restauración en Colombia Un compromiso de país, Bogotá: Editorial de la Red Colombiana de Restauración Ecológica, 2018.

[17] M. Swaine, Ed, "Functional Morphology of Tropical Tree Seedlings", de The Ecology of Tropical Forest Tree Seedling, Paris, UNESCO, 1996, pp. 56-129.

[18] L. v. d. Pijl, Principles of Dispersal in Higher Plants, New York: Springer Verlag, 1982.

[19] T. Gomes Calaça, M. J. Nogueira Rodal, A. L. Alves de Lima, L. Rodrigues Lima, M. Alves Diniz S. C. Pinto y A. Laurênio de Melo, "Do seedling functional groups reflect ecological strategies of woody plant species in Caatinga", Acta Botanica Brasilisca, vol. 32, no 1, pp. 20-27, 2018.

[20] M. Martínez-Ramos, E. Alvarez-Buylla y J. Sarukhán, "Tree Demography and Gap Dynamics in a Tropical Rain Forest", Ecology, vol. 70, n 3, pp. 555-558, 1989.

[21] T. C. Whitmore, "Canopy Gaps and the Two Major Groups of Forest Trees", Ecology, vol. 70, no 3, pp. 536-538, 1989.

[22] A. Martínez-Ramos, "Claros, ciclos vitales de los árboles tropicales y regeneración natural de las selvas altas perennifolias", de Investigaciones sobre la regeneración de selvas altas en Veracruz, Méxica, México, Alhambra Mexicana, 1895, p. 191-239.

[23] P. Y. Kageyama y V. M. Viana, "Tecnologia de sementes e grupos ecológicos de espécies 
Jorge A. Sierra-Escobar - Mario Alberto Quijano Abril - Daniela Marín-Henao Daniela Salazar Suaza - Mabel Vanessa García Garcés

arbóreas tropicais", de Simpósio Brasileiro sobre Tecnologia de Sementes Florestais, Atibai, 1989, pp. 197-215.

[24] G. Ibarra-Manríquez, M. Martinez-Ramos y K. Oyama, "Seedling Functional Types in a Lowland Rain Forest in Mexico", American Journal of Botany, vol. 88, $\mathrm{n}^{\circ}$ 10, pp. 180112,2001

[25] L. R. Holdridge, Ecología basada en zonas de vida, San José: Instituto Iberoamericano de cooperación para la agricultura (IICA), 2000.

[26] D. Jaramillo, "Introducción a la ciencia del suelo", Universidad Nacional de Colombia, sede Medellín, Medellín, 2002.

[27] ISA y JAUM, "Propuesta metológica de parcelas normalizadas para los inventarios de vegetación", Medellin, Antioquia. Colombia, 2001.

[28] "Análisis florístico y estructural de los bosques premontanos en el municipio de Amalfi", Colombia Forestal, vol. 12, n 1 , pp. 81-102, 2009.

[29] CORNARE, "Plan de ordenación y manejo de la microcuenca La Cristalina - La Risaralda. Municipio de San Luis", El Santuario, 2009.

[30] R. Liesner, C. Ulloa Ulloa y R. Ortiz, Técnicas de campo utilizadas por el Jardín Botánico de Missouri, Missouri Botanical Garden, 1996.

[31] O. Rangel Ch y G. Lozano, "Un perfil de vegetación entre La Plata (Huila) y el Volcán del Puracé", Caldasia, vol. 14, pp. 503-547, 1986.

[32] H. M. Villareal, M. Álvarez, S. CórdobaCórdoba, F. Escobar, G. Fagua, F. Gast, H. Mendoza-Cifuentes, M. Ospina y A. M. Umaña, Manual de metodos para el desarrollo de inventarios de biodiversidad, Bogotá: Instituto de Investigación de Recursos Biológicos Alexander von Humboldt, 2004.

[33] J. T. Curtis y R. P. McIntosh, "The Interrelations of Certain Analytic and Synthetic Phytosociological Characters", Ecology, vol. 31, n 3, pp. 434-455, 1950.

[34] U. Lüttge, M. Kluge y G. Bauer, Botánica, Madrid: McGraw-Hill, 1993.
[35] A. Jiménez-Valverde y J. Hortal, "Las curvas de acumulación de especies y la necesidad de evaluar la calidad de los inventarios biológicos", Revista Ibérica de Aracnología, vol. 8, pp. 151-161, 2002.

[36] E. Gangenova, A. Guzmán y F. Marangoni, "Diversidad de anfibios anuros del Parque Nacional El Palmar (Provincia de Entre Ríos, Argentina)", Revista de la Asociación Herpetológica de Argentina, vol. 26, n 1, 2012.

[37] Y. G. Ocaña Figueroa, "Caracterización florística y estructural de unidades de bosque altoandino en las Veredas Minas y Patios Altos, Enciso - Santander", Colombia Forestal, vol. 9, no 18, pp. 70-86, 2005.

[38] E. Calderón, G. Galeano y N. García, Libro rojo de plantas de Colombia. Vol 2: Palmas, frailejones y zamias. Serie Libros rojos de especies amenazadas, Bogotá: Instituto Alexander von Humbolt, 2005.

[39] J. Orellana Lara, Determinación de indices de diversidad florística arbórea en las parcelas permanentes de muestreo del valle de Sacta, Cochabamba, Bolivia: Universidad Mayor de San Simon, 2009.

[40] O. Vargas, J. E. Díaz, S. Reyes y P. A. GómezRuiz, Guías técnicas para la restauración ecológica de los ecosistemas de Colombia, Bogotá: Universidad Nacional de Colombia, 2012.

[41] J. Carabias, J. A. Meave, T. Valverde y Z. Cano-Santana, Ecología y medio ambiente en el siglo XXI, Ciudad de México: Pearson Educación, 2009.

[42] M. Reina, R. Medina, F. A. Ávila, S. P. Ángel y R. Cortés B, "Catálogo preliminar de la flora vascular de los bosques subandinos de la reserva biológica Cachalú, Santander (Colombia)", Colombia Forestal, vol. 13, $\mathrm{n}^{\mathrm{o}}$ 1, pp. 27-54, 2010.

[43] A. Rincon Velásquez, M. A. Triana Gómez y J. . D. Burgos, "Caracterización florística y estructural de la vegetación en claros de la parcela permanente de 25 ha en la reserva natural La Planada (Nariño)", Colombia Forestal, vol. 9, n 19, pp. 5-29, 2006.

[44] J. I. Barrera Cataño, S. M. Contreras Rodríguez, N. V. Garzón Yepes y A. C. Moreno Cárdenas, Manual para la Restauración 
Jorge A. Sierra-Escobar - Mario Alberto Quijano Abril - Daniela Marín-Henao Daniela Salazar Suaza - Mabel Vanessa García Garcés

Ecológica de los Ecosistemas Disturbados del Distrito Capital, Bogotá: Secretaría Distrital de Ambiente (SDA), Pontificia Universidad Javeriana (PUJ, 2010.

[45] E. Cantillo Higuera y M. Gracia Cuellar, "Diversidad y caracterización florística de la vegetación natural en tres sitios de los cerros orientales de Bogotá D. C.", Colombia Forestal, vol. 16, nº 2, pp. 228-256, 2013.

[46] A. R. Rodríguez-Lombana, H. E. BeltránGutiérrez y A. . C. Moreno, "Caracterización florística del bosque subandino y algunas áreas disturbadas en San Bernardo (Cundinamarca), Colombia", Biota Colombiana, vol. 18, n 2, pp. 42-71, 2017.

[47] E. Ceccon, Restauración en bosques tropicales: fundamentos ecológicos, prácticos y sociales, México: Ediciones Díaz de Santos, 2013.

[48] S. Elliotta, P. Navakitbumrung, C. Kuarak, S. Zangkum, V. Anusarnsunthorn y D. Blakesley, "Selecting framework tree species for restoring seasonally dry tropical forests in northern Thailand based on field performance", Forest Ecology and Management, vol. 184, p. 177-191, 2013.

[49] P. Y. Kageyama, R. E. Oliveira, L. Moraes, V. L. Engel y F. B. Gandara, Restauração de áreas degradadas utilizando interação interespecíficas, Botucatu: FEPAF, 2003.

[50] K. Holl, "Prioridades de Investigación en Restauración de Bosques Neotropicales", de Restauración Ecológica en Colombia "Un compromiso de pais", Bogotá, Red Colombiana de Restauración Ecológica, 2018, p. 418.

[51] F. Torres Romero, L. A. Villota Ojeda y J. C. Villalba Malaver, "Avances en domesticación de especies nativas con potencial para los procesos de restauración ecológica", de Restauración Ecológia en Colombia "Un compromiso de país", Bogotá, Red Colombiana de Restauración Ecológica, Universidad Católica de Oriente, 2018, p. 418.

[52] F. Álzate y J. Sierra-Escobar, Metodología para la selección de especies en un programa de fuentes semilleras, Rionegro: Universidad Católica de Oriente, 2000.

[53] F. P. Melo, S. R. Pinto, P. H. Brancalio, P. S. Castro, R. S. Rodrigues, J. Aronson y M. Tabarelli, "Priority setting for scaling. up tropical forest restoration projetcs: Early lessons from the Atlantic forest restoration pact", Enviromental Science \& Policy, vol. 33, pp. 395-404, 2013. 